\title{
C278F mutation in FGFR2 gene causes two different types of syndromic craniosynostosis in two Chinese patients
}

\author{
YING LIN $^{1^{*}}$, HONGBIN GAO ${ }^{2,3^{*}}$, SIMING AI $^{1^{*}}$, JACOB V.P. ESWARAKUMAR ${ }^{4 *}$, CHUAN CHEN $^{1,5}$, \\ YI ZHU ${ }^{1,5}$, TAO LI ${ }^{1}$, BINGQIAN LIU ${ }^{1}$, XIALIN LIU ${ }^{1}$, LIXIA LUO ${ }^{1}$, HONGYE JIANG $^{6}$, YONGHAO LI ${ }^{1}$, \\ XIAOLING LIANG ${ }^{1}$, CHENJIN JIN $^{1}$, XINHUA HUANG ${ }^{1 *}$ and LIN LU ${ }^{1 *}$ \\ ${ }^{1}$ State Key Laboratory of Ophthalmology, Zhongshan Ophthalmic Center, Sun Yat-sen University, Guangzhou, \\ Guangdong 510060; ${ }^{2}$ Guangdong Laboratory Animals Monitoring Institute, Key Provincial Laboratory of Guangdong \\ Laboratory Animals, Guangzhou, Guangdong 510663; ${ }^{3}$ Department of Toxicology, School of Public Health and \\ Tropical Medicine, Southern Medical University, Guangzhou, Guangdong 510515, P.R. China; ${ }^{4}$ Department of \\ Pharmacology, Yale University School of Medicine, New Haven, CT 06520; ${ }^{5}$ Department of Molecular and Cellular \\ Pharmacology, University of Miami Miller School of Medicine, Miami, FL 33136, USA; ${ }^{6}$ Department of Obstetrics \\ and Gynecology, The First Affiliated Hospital, Sun Yat-sen University, Guangzhou, Guangdong 510000, P.R. China
}

Received September 18, 2016; Accepted May 24, 2017

DOI: $10.3892 / \mathrm{mmr} .2017 .7248$

\begin{abstract}
The current study was performed with aim to investigate the fibroblast growth factor receptor 2 (FGFR2) gene in two Chinese families with two different forms of syndromic craniosynostosis, and to characterize their associated clinical features. Two families underwent complete ophthalmic examinations, and two patients from each family were diagnosed with craniosynostosis. Genomic DNA was extracted from leukocytes of peripheral blood collected from these two families and from 200 unrelated subjects within the same population as controls. Exons 8 and 10 of the FGFR2 gene were amplified by polymerase chain reaction and directly sequenced. Ophthalmic examinations of the two patients revealed shallow orbits and ocular proptosis, accompanied by midface hypoplasia and craniosynostosis. Case 1 had retinal detachment, abnormal limbs and hands, while case 2 exhibited normal hands and feet upon clinical examination. A heterozygous FGFR2 missense mutation c.833G>T (C278F) in exon 8 was identified in these two patients, but not in unaffected family members or the normal controls. Although FGFR2 gene mutations and polymorphisms have been studied in various ethnic groups, we report a mutation of FGFR2 in
\end{abstract}

Correspondence to: Professor Xinhua Huang or Professor Lin Lu, State Key Laboratory of Ophthalmology, Zhongshan Ophthalmic Center, Sun Yat-sen University, 54 Xianlie South Road, Guangzhou, Guangdong 510060, P.R. China

E-mail: 1476557150@qq.com

E-mail: drlulin@126.com

*Contributed equally

Key words: craniosynostosis, fibroblast growth factor receptor 2, gene, mutation, Peters anomaly, Pfeiffer syndrome, Crouzon syndrome two different Chinese patients with two different types of syndromic craniosynostosis.

\section{Introduction}

Craniosynostosis is a group of connective disorders that primarily presents with premature fusion of cranial sutures, and has a birth prevalence of 1 in 2,000-3,000 live births $(1,2)$. Many syndromes associated with craniosynostosis have been studied, the majority of which exhibit dominant inheritance (3). These syndromes are clinically classified according to the presence of specific craniofacial abnormalities with or without specific limb involvement $(2,4)$. Among these autosomal dominant forms of the craniofacial complex, Crouzon syndrome is the most distinctive and common disorder characterized by the triad of premature craniosynostosis, orbital proptosis and midfacial hypoplasia $(5,6)$. Historically, Crouzon syndrome was distinguished clinically by the absence of limb defects. Sometimes, it is a difficult diagnostic for clinicians $(7,8)$. Since clinical classification of craniosynostosis syndromes is challenging, investigations of the underlying gene mutations have great value for differentiation of these disorders (9), and may help to determine the molecular basis of the syndrome.

The molecular analysis of fibroblast growth factor receptor $(F G F R)$ genes is of great value in the clinical by providing confirmatory diagnosis, and also allows prenatal diagnosis (10). FGFRs are transmembrane signal-transduction molecules. Upon binding of fibroblast growth factors (FGFs) to their extracellular region, FGFRs dimerize to induce trans-autophosphorylation of the intracellular tyrosine kinase region $(11,12)$. The sequence structure of FGFRs share great similarity, which is characterized by three extracellular immunoglobulin-like domains (IgI, IgII and IgIII), a single transmembrane domain and a split tyrosine kinase (TK1/TK2) domain. FGFR2 gene is located at 10q26, and 
$\sim 95 \%$ of cases have mutations in exons IIIa (exon 8) and IIIc (exon 10) (13).

The present study performed mutational analysis of two Chinese families with craniosynostosis syndrome at the gene level, and identified one recurrent heterozygous mutation and characterized its associated clinical features.

\section{Patients and methods}

Ethics. All experimental protocols and the methods were performed in accordance with the standard guidelines and approved by the Ethics Committee of Zhongshan Ophthalmic Center (Guangzhou, China). Written informed consent, for participation in the present study and the publication of images, was obtained from all participating subjects in accordance with the Declaration of Helsinki.

A Crouzon syndrome family. Two probands in two Chinese families were diagnosed with craniosynostosis at Zhongshan Ophthalmic Center. The proband of family 1 (Fig. 1) was a 53-year old man, whose sister did not have craniosynostosis. The proband of family 2 (Fig. 2) was a 7-year old girl who was the only child of her family. Ophthalmic examinations were performed in these two families, as follows: Visual acuity was tested using the Early Treatment Diabetic Retinopathy Study chart (Precision Vision, Woodstock, IL, USA); anterior segment photographs were captured using the BX 900 Slit Lamp (Haag-Streit AG, Köniz, Switzerland); fundus photography was performed using a Heidelberg Retina Angiograph (Heidelberg Engineering GmbH, Heidelberg, Germany); computed tomography (CT) and physical examinations, including blood and urine tests, electrocardiogram, X-ray, blood biochemistry test, blood lipid and blood coagulation tests were conducted to exclude systemic diseases.

Sample collection. The affected families were identified at Zhongshan Ophthalmic Center. As a normal control, two hundred subjects without diagnostic features of craniosynostosis syndrome from the same population were recruited. Venous blood samples were collected, and genomic DNA was extracted from peripheral blood leucocytes using standard protocols and a DNA extraction kit (Qiagen $\mathrm{GmbH}$, Hilden, Germany). Patients with primary microcephaly, postural plagiocephaly, incomplete data, no visual perception or lost from follow-up were excluded from the study.

Mutation detection. Exons 8 and 10 of the FGFR2 gene were amplified by polymerase chain reaction (PCR) with previously established primers $(11,14,15)$. Briefly, PCR was performed in $50 \mu \mathrm{l}$ reactions using the PCR Amplification kit (Takara Bio, Inc., Otsu, Japan). The cycling profile was initiated by one cycle at $94^{\circ} \mathrm{C}$ for $5 \mathrm{~min}$, followed by 40 cycles at $94^{\circ} \mathrm{C}$ for $45 \mathrm{sec}$, $52-66^{\circ} \mathrm{C}$ for $45 \mathrm{sec}$ and $72^{\circ} \mathrm{C}$ for $45 \mathrm{sec}$, and ended with one cycle at $72^{\circ} \mathrm{C}$ for $10 \mathrm{~min}$. The PCR products were sequenced from both directions with an ABI3730 Automated Sequencer (Thermo Fisher Scientific, Inc., Waltham, MA, USA). The sequencing results were analyzed using Chromas (version 2.3; Technelysium Pty Ltd, Brisbane, Australia) by comparing with the reference sequences in the database at the National Center for Biotechnology Information (NCBI; NC_000010).

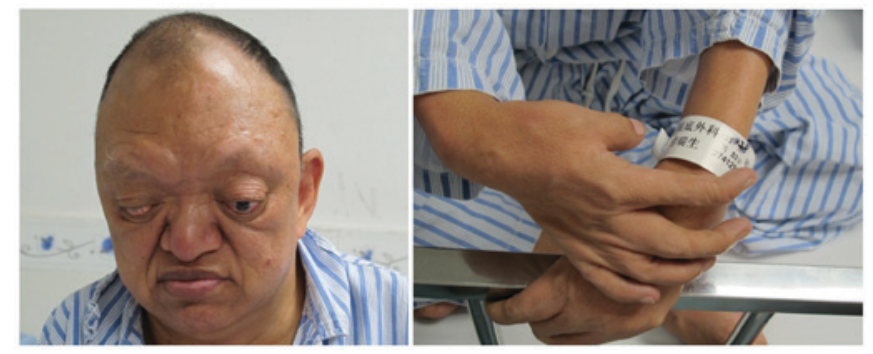

Figure 1 . The proband of family 1 was a 53 -year-old man with craniosynostosis. The patient had shallow orbits and ocular proptosis, accompanied by midface hypoplasia, craniosynostosis with clinically abnormal hands and feet, without broad thumbs.

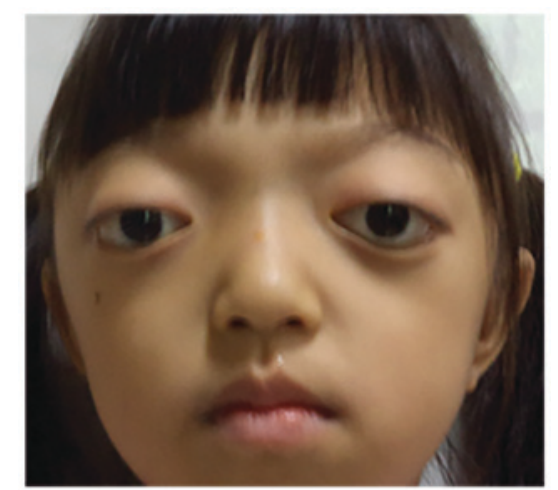

Figure 2. The second proband of family 2 was a 7-year-old girl with craniosynostosis, and she had shallow orbits and ocular proptosis, accompanied by midface hypoplasia, craniosynostosis with clinically normal hands and feet.

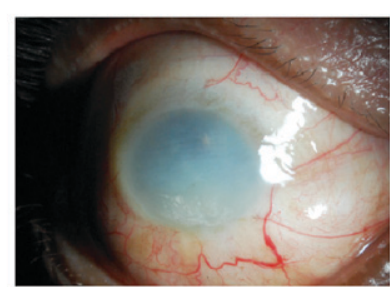

OD

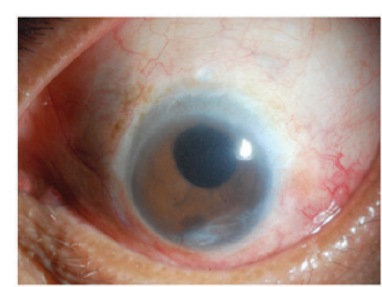

os
Figure 3. Eye abnormalities in case 1 . The cornea was small with a diameter of $9 \mathrm{~mm}$ and fully white in the right eye. The left eye experienced retinal detachment 2 years ago, and had been operated on with vitrectomy, cataract surgery and silicone oil implantation. Microcornea and absent lens are observed. OS, oculus sinister; OD, oculus dextrus.

\section{Results}

Clinical data. The Chinese families studied in this project were from the southern area of China. Two individuals, in two separate families, were identified as having similar disease and other systemic diseases were excluded (data not shown). These patients exhibited from shallow orbits and ocular proptosis, accompanied by midface hypoplasia, craniosynostosis and a curved, beaklike nose. Besides these manifestations, case 1 in family 1 had retinal detachment and clinically abnormal hands and feet, without broad thumbs (Fig. 1).

The best-corrected visual acuity of case 1 as measured using a LogMAR chart. The visual acuity was no light 


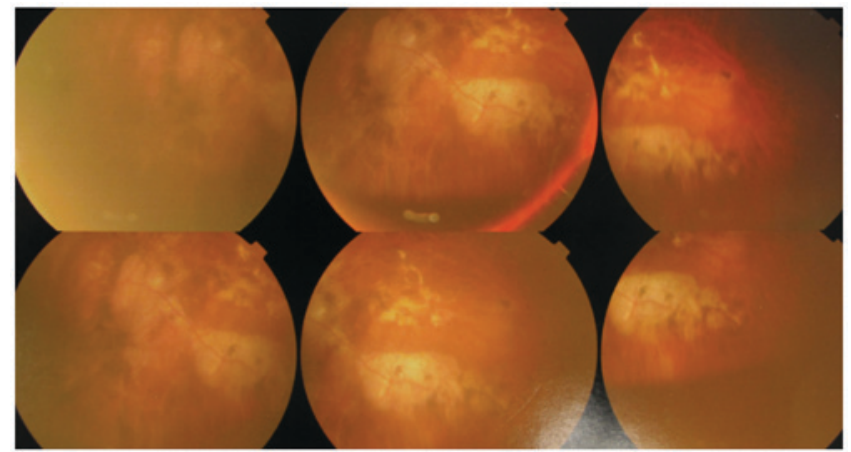

Figure 4. Retinal atrophy in fundus photographs of case 1.

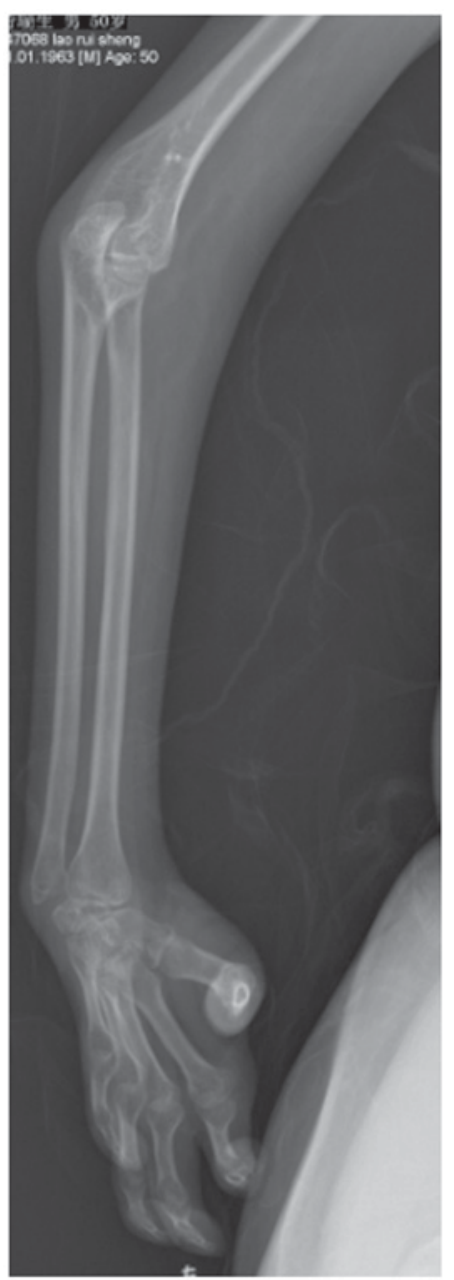

Figure 5. X-ray demonstrated abnormal limbs of case 1.

perception of the right eye (oculus dextrus; OD) and counting figure/50CM of the left eye (oculus sinister; OS). The patient could see nothing when he was born due to congenital ocular abnormalities. The diameter of the cornea was $\sim 9 \mathrm{~mm}$ and the cornea was completely white, obscuring the lens and the fundus (Fig. 3A). Retinal detachment occurred 2 years ago, and had been treated with vitrectomy, cataract surgery and silicone oil implantation, so the lens could not be observed (Fig. 3B). Some retinal atrophy was observed in the fundus photographs (Fig. 4) and the intraocular pressure was $34 \mathrm{mmHg}$. X-ray showed abnormal limbs (Fig. 5).
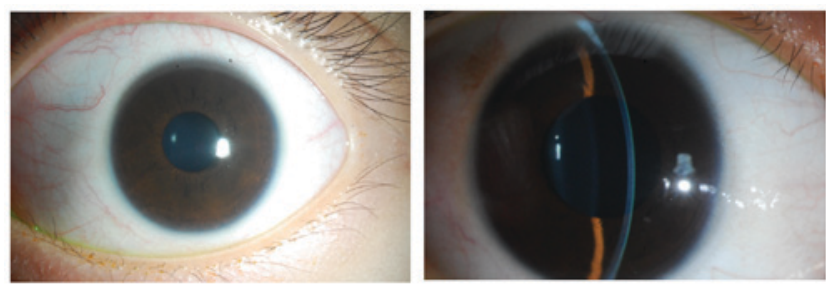

Figure 6. Images of the eyes of case 2. The anterior segment photograph demonstrated that the corneas were normal in size and transparency, and the lenses were positioned normally and remained clear.
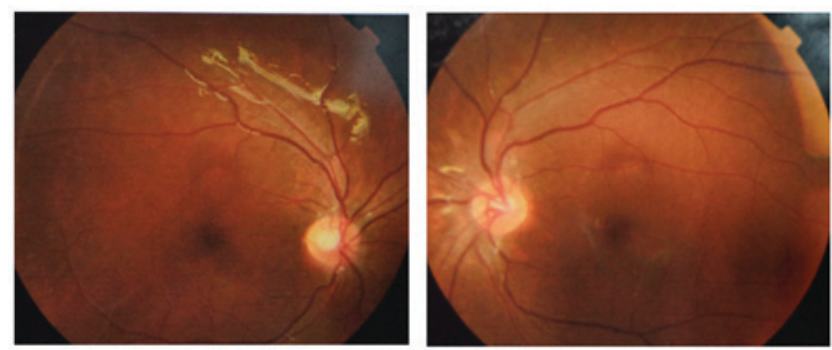

Figure 7. No abnormalities were detected in the retinas, choroids, or optic nerves of the fundus photographs of case 2 .

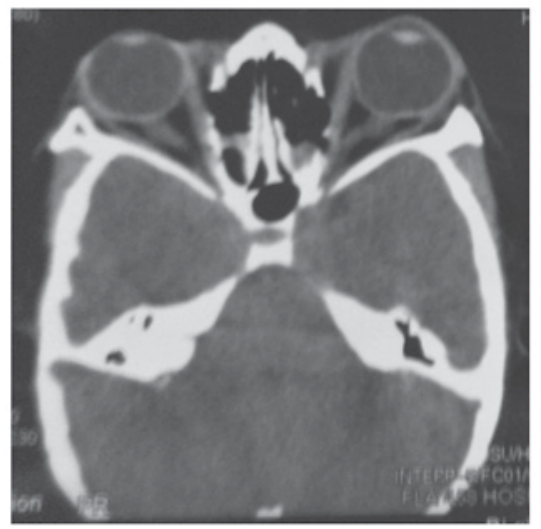

Figure 8. Computed tomography of the skull of the patient was performed and revealed shallow orbits in case 2 .

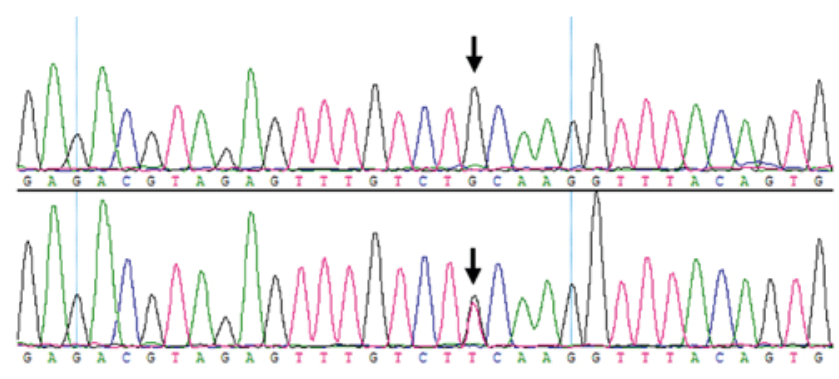

Figure 9. A heterozygous fibroblast growth factor receptor 2 missense mutation c.833G $>$ T $(C 278 F)$ in exon 8 was identified in the affected individuals (cases 1 and 2), but not in any of the unaffected family members and the normal controls. The mutation causes the cysteine 278 codon (TGC) to change to a phenylalanine codon (TTC).

The best-corrected visual acuity of case 2 in family 2 was measured by LogMAR, and was $0.00(\mathrm{OD})$ and $0.10(\mathrm{OS})$. The results of the optometry were -0.50DS (OD) and +3.00DS 
(OS). Applanation tonometry revealed normal bilateral intraocular pressure. The corneas were transparent and normal in size, and the lenses were clear and were positioned normally (Fig. 6). No abnormalities were found in the retinas, choroids or optic nerves (Fig. 7). CT of the skull of this patient (Fig. 8) revealed shallow orbits.

Mutation screening. A heterozygous FGFR2 missense mutation c. $833 \mathrm{G}>\mathrm{T}(\mathrm{C} 278 \mathrm{~F})$ in exon 8 was identified in both affected individuals, but not in any of the unaffected family members or the normal controls. The mutation causes a change from the cysteine 278 codon (TGC) to a phenylalanine codon (TTC; Fig. 8). As the parents of case 1 had been dead for several years, it cannot be determined whether the mutation was inherited. The parents of Case 2 had no mutations with normal appearance, therefore, case 2 was caused by a de novo mutation.

\section{Discussion}

The current study identified a mutation of the FGFR2 gene that is associated with craniosynostosis syndrome: c. $833 \mathrm{G}>\mathrm{T}$ $(\mathrm{C} 278 \mathrm{~F})$ in exon 8 . This mutation is the causative mutation in two patients with craniosynostosis syndrome from two different families, rather than a rare polymorphism in the normal population.

Craniosynostosis are a group of connective disorders characterized by premature fusion of cranial sutures. Three clinically associated craniosynostosis, Crouzon, Jackson-Weiss (JW) and Pfeiffer syndromes (PS), share common clinical features including craniosynostosis, ocular hypertelorism with proptosis and midface hypoplasia $(10,16)$. The craniosynostosis syndromes differ by the absence of limb abnormalities in Crouzon syndrome and presence of limb abnormalities in PS and JW. Previously, clinical distinction between PS and JW was determined by the presence of broad great toes with medial deviation and tarsal-metatarsal coalescence without hand anomalies in JW $(9,17)$. Craniosynostosis is associated with a variety of ophthalmic abnormalities. For example, patients with craniosynostosis may have anterior segment dysgenesis and present Peters anomaly, which is characterized by microphthalmia, microcornea, sclerocornea, glaucoma, chorioretinal coloboma, corneal perforation, and retinal detachment (9). Case 1 in the present study presented with retinal detachment and microcornea. Some cases of Peters anomaly without systemic complications have an autosomal dominant or recessive inheritance pattern. Cases with short limb dwarfism are designated as Peters-plus syndrome, which is currently believed to be autosomal recessive. Additionally, Peters anomaly has been reported in Pfeiffer syndrome $(9,17)$. Therefore, case 1 was diagnosed as Pfeiffer syndrome with Peters-plus syndrome and case 2 was diagnosed as Crouzon syndrome. Surprisingly, the same FGFR2 mutation can lead to CS, PS or JW phenotype $(8,18,19)$.

To date, $\sim 16$ genes associated with craniosynostosis have been identified. Although craniosynostosis can be classified into several different syndromes based on clinical phenotypes, correlation between genotype and phenotype has not yet been established. In the majority of cases, pathogenic FGFR mutations are missense, which all confer gain-of-function to the mutated protein; some mutations are highly recurrent $(10,20)$. The c. $833 \mathrm{G}>\mathrm{T}(\mathrm{C} 278 \mathrm{~F})$ was identified in the FGFR2 gene in two different Chinese patients with different kinds of syndromic craniosynostosis in the current study; however, it occurred at a hotspot for mutation, which has been reported in other ethnic groups $(17,18)$.

It is clear that the same mutation may result in heterogeneous phenotypes, as the $\mathrm{C} 278 \mathrm{~F}$ mutation in $F G F R 2$ can lead to Crouzon syndrome and Pfeiffer syndrome. The mechanism by which the same genotype causes different phenotypes remains unclear. One potential explanation may be due to other unidentified polymorphisms within FGFR2. Cys 278 is part of the disulfide bridge that stabilizes the IgIII loop in all FGFR proteins and is the most conserved amino acid of the extracellular domains in the Ig superfamily. The loss of Cys 278 destroys the disulfide bond in the Ig-III domain, leaving the other cysteine at position 342 potentially unpaired to participate in intermolecular disulfide bonding, which potentially results in a ligand-independent dimerization of receptor molecules and, thus, constitutive activation $(21,22)$. Similar hypotheses apply to mutations affecting Cys 342 in other Crouzon syndrome patients $(23,24)$. Mutations of either Cys 342 or Cys 278 can cause a severe craniosynostosis phenotype. In addition, although the same $\mathrm{C} 278 \mathrm{~F}$ mutation in FGFR2 can lead to different types of craniosynostosis, the treatments are similar and surgery is usually required in severe cases (25-27).

In summary, this study identified an FGFR2 in two Chinese patients with syndromic craniosynostosis. The finding expands the reported mutation spectrum of FGFR2, and is of great value for genetic counseling and prenatal diagnosis in families with syndromic craniosynostosis.

\section{Acknowledgements}

This study was supported by the National Natural Science Foundation of China (grant nos. 81500709, 81570862, 81670872 and 81371019) and the Medical Scientific Research Foundation of Guangdong Province (grant no. A2016460).

\section{References}

1. Dicus Brookes C, Golden BA and Turvey TA: Craniosynostosis syndromes. Atlas Oral Maxillofac Surg Clin North Am 22: 103-110, 2014.

2. Buchanan EP, Xue AS and Hollier LH Jr: Craniofacial syndromes. Plast Reconstr Surg 134: 128e-153e, 2014.

3. Cohen MM Jr: Craniosynostosis update 1987. Am J Med Genet Suppl 4: 99-148, 1988.

4. Bowling EL and Burstein FD: Crouzon syndrome. Optometry 77: 217-222, 2006.

5. Giordano BP, Tuli SS, Ryan SF, Stern M and Tuli SY: Crouzon Syndrome: Visual Diagnosis. J Pediatr Health Care 30: 270-273, 2016.

6. Gorry MC, Preston RA, White GJ, Zhang Y, Singhal VK, Losken HW, Parker MG, Nwokoro NA, Post JC and Ehrlich GD: Crouzon syndrome: Mutations in two spliceoforms of FGFR2 and a common point mutation shared with Jackson-Weiss syndrome. Hum Mol Genet 4: 1387-1390, 1995.

7. Khandelwal R, Agrawal P and Majumdar MR: Crouzon syndrome. BMJ Case Rep 2012: pii: bcr0520114196, 2012.

8. Mitulla B, Hinkel GK and Lorenz P: Crouzon syndrome (Mc K 12350). Kinderarztl Prax 59: 278-280, 1991.

9. Okajima K, Robinson LK, Hart MA, Abuelo DN, Cowan LS, Hasegawa T, Maumenee IH and Jabs EW: Ocular anterior chamber dysgenesis in craniosynostosis syndromes with a fibroblast growth factor receptor 2 mutation. Am J Med Genet 85: 160-170, 1999. 
10. Schaefer F, Anderson C, Can B and Say B: Novel mutation in the FGFR2 gene at the same codon as the Crouzon syndrome mutations in a severe Pfeiffer syndrome type 2 case. Am J Med Genet 75: 252-255, 1998.

11. Lin Y, Liang X, Ai S, Chen C, Liu X, Luo L, Ye S, Li B, Liu Y and Yang H: FGFR2 molecular analysis and related clinical findings in one Chinese family with Crouzon syndrome. Mol Vis 18 449-454, 2012.

12. Tan X, Zhu Y, Chen C, Chen X, Qin Y, Qu B, Luo L, Lin H, Wu M, Chen W and Liu Y: Sprouty2 suppresses epithelial-mesenchymal transition of human lens epithelial cells through blockade of Smad2 and ERK1/2 Pathways. PLoS One 11: e0159275, 2016.

13. Kan SH, Elanko N, Johnson D, Cornejo-Roldan L, Cook J, Reich EW, Tomkins S, Verloes A, Twigg SR, Rannan-Eliya S, et al: Genomic screening of fibroblast growth-factor receptor 2 reveals a wide spectrum of mutations in patients with syndromic craniosynostosis. Am J Hum Genet 70: 472-486, 2002.

14. Lin Y, Ai S, Chen C, Liu X, Luo L, Ye S, Liang X, Zhu Y, Yang H and Liu Y: Ala344Pro mutation in the FGFR2 gene and related clinical findings in one Chinese family with Crouzon syndrome. Mol Vis 18: 1278-1282, 2012

15. Lin Y, Liu X, Yu S, Luo L, Liang X, Wang Z, Chen C, Zhu Y, Ye S, Yan H and Liu Y: PAX6 analysis of two sporadic patients from southern China with classic aniridia. Mol Vis 18: 2190-2194, 2012.

16. Shotelersuk V, Mahatumarat C, Ittiwut C, Rojvachiranonda N, Srivuthana S, Wacharasindhu S and Tongkobpetch S: FGFR2 mutations among Thai children with Crouzon and Apert syndromes. J Craniofac Surg 14: 101-107, 2003.

17. Robin NH, Falk MJ, Haldeman-Englert CR: FGFR-Related Craniosynostosis Syndromes. In: Pagon RA, Adam MP, Ardinger HH, Wallace SE, Amemiya A, Bean LJH (eds), et al: GeneReviews (R). Seattle (WA), 1993.

18. Murano I: Crouzon syndrome. Nihon Rinsho 28 (Suppl 3): S416-S417, 2006 (In Japanese).

19. Mehta P, Robson CN, Neal DE and Leung HY: Fibroblast growth factor receptor- 2 mutation analysis in human prostate cancer. BJU Int 86: 681-685, 2000.
20. Snyder-Warwick AK, Perlyn CA, Pan J, Yu K, Zhang L and Ornitz DM: Analysis of a gain-of-function FGFR2 Crouzon mutation provides evidence of loss of function activity in the etiology of cleft palate. Proc Natl Acad Sci USA 107: 2515-2520, 2010.

21. Ke R, Yang X, Tianyi C, Ge M, Lei J and Mu X: The C342R mutation in FGFR2 causes Crouzon syndrome with elbow deformity. J Craniofac Surg 26: 584-586, 2015.

22. Ratisoontorn C, Fan GF, McEntee K and Nah HD: Activating (P253R, C278F) and dominant negative mutations of FGFR2: Differential effects on calvarial bone cell proliferation, differentiation, and mineralization. Connect Tissue Res 44 (Suppl 1): S292-S297, 2003.

23. Kan SH, Elanko N, Johnson D, Cornejo-Roldan L, Cook J, Reich EW, Tomkins S, Verloes A, Twigg SR, Rannan-Eliya S, et al: Genomic screening of fibroblast growth-factor receptor 2 reveals a wide spectrum of mutations in patients with syndromic craniosynostosis. Am J Hum Genet 70: 472-486, 2002.

24. Lajeunie E, Heuertz S, El Ghouzzi V, Martinovic J, Renier D, Le Merrer M and Bonaventure J: Mutation screening in patients with syndromic craniosynostoses indicates that a limited number of recurrent FGFR2 mutations accounts for severe forms of Pfeiffer syndrome. Eur J Hum Genet 14: 289-298, 2006.

25. Mangasarian K, Li Y, Mansukhani A and Basilico C: Mutation associated with Crouzon syndrome causes ligand-independent dimerization and activation of FGF receptor-2. J Cell Physiol 172: 117-125, 1997.

26. Reardon W, Winter RM, Rutland P, Pulleyn LJ, Jones BM and Malcolm S: Mutations in the fibroblast growth factor receptor 2 gene cause Crouzon syndrome. Nat Genet 8: 98-103, 1994.

27. Sagong B, Jung DJ, Baek JI, Kim MA, Lee J, Lee SH, Kim UK and Lee KY: Identification of causative mutation in a Korean family with Crouzon syndrome using whole exome sequencing. Ann Clin Lab Sci 44: 476-483, 2014. 